\title{
Principles and practice of functional MRI of the human brain
}

\author{
John C. Gore \\ Vanderbilt University Institute of Imaging Science, Nashville, Tennessee, USA \\ J. Clin. Invest. 112:4-9 (2003). doi:10.1172/JCI200319010.
}

Conventional MRI is used extensively for radiological diagnosis and produces spatial maps of the properties of mobile hydrogen nuclei (single protons) that are contained mainly in water molecules. Conventional magnetic resonance images portray anatomic details with exquisite resolution (on the order of $1 \mathrm{~mm}$ or better), in three dimensions, and differentiate soft tissues very well. The contrast within images results from variations mainly in the density of water within tissues and in the manner in which water interacts with macromolecules. Functional MRI (fMRI) is now also well established in many centers and uses similar imaging techniques and the same equipment as conventional MRI. PMRI relies on detecting small changes in the signals used to produce magnetic resonance images that are associated with neuronal activity in the brain, and it is producing unique and valuable information for applications in both basic and clinical neuroscience. PMRI is safe, noninvasive, and repeatable in adults and children and thus has widespread potential uses. This short overview will attempt to illustrate the physical basis of fMRI, how it is performed in practice, some of the limitations of the technique, and some of the types of application that have been exploited to date.

fMRI detects the blood oxygen level-dependent (BOLD) changes in the MRI signal that arise when changes in neuronal activity occur following a change in brain state, such as may be produced, for example, by a stimulus or task. One of the underlying premises of many current uses of functional imaging is that various behaviors and brain functions rely on the recruitment and coordinated interaction of components of "largescale" brain systems that are spatially distinct, distributed, and yet connected in functional networks. Thus, although the practice of phrenology is dead, identification of the neurobiological substrates associated with various specific functions of the brain is likely to shed light on how the brain determines behavior. In addition,

Address correspondence to: John C. Gore, Vanderbilt University Institute of Imaging Science, MCN R1302, 1161 21st Avenue South, Nashville, Tennessee 37232, USA. Phone: (615) 322-8357; Fax: (615) 322-0734; E-mail: john.gore@vanderbilt.edu.

Conflict of interest: The author has declared that no conflict of interest exists.

Nonstandard abbreviations used: functional MRI (fMRI); blood oxygen level-dependent (BOLD). geographic maps identifying the locations of particularly critical areas, such as those involved in producing and understanding language, are of direct importance in clinical assessments and the planning of interventions.

The physical origins of BOLD signals are reasonably well understood, though their precise connections to the underlying metabolic and electrophysiological activity need to be clarified further. It is well established that an increase in neural activity in a region of cortex stimulates an increase in the local blood flow in order to meet the larger demand for oxygen and other substrates. The change in blood flow actually exceeds that which is needed so that, at the capillary level, there is a net increase in the balance of oxygenated arterial blood to deoxygenated venous blood. Essentially, the change in tissue perfusion exceeds the additional metabolic demand, so the concentration of deoxyhemoglobin within tissues decreases. This decrease has a direct effect on the signals used to produce magnetic resonance images. While blood that contains oxyhemoglobin is not very different, in terms of its magnetic susceptibility, from other tissues or water, deoxyhemoglobin is significantly paramagnetic (like the agents used for MRI contrast materials, such as gadolinium), and thus deoxygenated blood differs substantially in its magnetic properties from surrounding tissues (1). When oxygen is not bound to hemoglobin, the difference between the magnetic field applied by the MRI machine and that experienced close to a molecule of the blood protein is much greater than when the oxygen is bound. On a microscopic scale, replacement of deoxygenated blood by oxygenated blood makes the local magnetic environment more uniform. The longevity of the signals used to produce magnetic resonance images is directly dependent on the uniformity of the magnetic field experienced by water molecules: the less uniform the field, the greater the mixture of different signal frequencies that arise from the sample, and therefore the faster the decay of the overall signal.

The result of having lower levels of deoxyhemoglobin present in blood in a region of brain tissue is therefore that the MRI signal from that region decays less rapidly and so is stronger when it is recorded in a typical magnetic resonance image acquisition. This small signal increase is the BOLD signal recorded in FMRI (Figure 1) (2). It is typically around $1 \%$ or less, though it varies 
depending on the strength of the applied field; this variability is one reason why higher-field MRI systems are being developed. As can be predicted from the above explanation, the magnitude of the signal depends on the changes in blood flow and volume within tissue, as well as the change in local oxygen tension, so there is no simple relation between the signal change and any single physiological parameter. Thus fMRI does not report absolute changes such as the units of flow obtained with positron emission tomographic (PET) imaging. Furthermore, as neurons become more active, there is a time delay before the necessary vasodilation can occur to increase flow, and for the wash-out of deoxyhemoglobin from the region to occur. Thus the so-called hemodynamic response detected by BOLD imaging is delayed and has a duration of several seconds following a stimulating event (3).

In AMRI, a subject is placed in the magnet of an MRI machine, where various different kinds of stimulus may be administered in a controlled fashion. For example, sounds may be played, visual scenes may be presented, and small motor movements or responses can be recorded. Although conventional imaging methods can be adapted for fMRI (4), most studies are performed using "snapshot" imaging methods, of which echo-planar imaging is the main exemplar (5). Improvements in gradient coil technology in recent years have permitted the implementation of such ultrafast imaging methods, in which complete cross-sectional images are recorded in substantially less than a second (typically about 50-100 ms). Although these images have poorer resolution and overall quality than the images used for radiological diagnosis, they do not suffer blurring from physiological motion, they permit multi-slice recording of the entire brain in a few seconds, and, in principle, they permit sampling of the hemodynamic response to transient events, as well as multiple recordings of repeated stimuli over a typical experimental time course. The images in a sequence may differ from one another even when there is no change in brain state, because of signal variance and the effects of extraneous "noise" (such as that produced by random voltages within the coils and components used to record MRI signals), so fMRI studies compare sets of images acquired during two or more different conditions using some form of statistical analysis. Multiple recordings of the MRI signal permit a degree of signal averaging that increases the reliability of the results.

Among the many types of study that can be performed, two main experimental paradigms are in common use. In so-called block designs, stimuli are presented in alternating short runs ("blocks") of several seconds' duration, and the MRI signals are then compared for the two types of blocks. For a visual-stimulation task to localize primary visual areas, a subject might view a bright flickering checkerboard for $20 \mathrm{sec}-$ onds, followed by a dark screen for 20 seconds, with these blocks repeated several times; eight pairs of blocks, for example, would require a total recording that lasts under 6 minutes. During that time, images may be recorded for many different parallel slices (typically 10-20), such that each slice is imaged about every 2 seconds. In this example, 80 images would be acquired for each slice for both conditions (stimulus ON and OFF). Those volume elements (voxels) within the brain that are affected by the stimulus (such as the primary visual cortex) provide a sequence of data points in which the signal alternates in intensity in synchrony with the stimulation because of the BOLD effect (Figure 2). By detection of which voxels show this alternating pattern, the visual cortex can be identified. The block design is simple to implement and can be used to localize several basic functions including: (i) primary sensory areas, e.g., by presenting auditory stimuli versus silence; (ii) areas involved with simple motor tasks, such as finger tapping; (iii) higher-order visual areas, for example, by presenting faces versus other objects to identify regions that respond preferentially to faces; and (iv) higher-order language areas by presenting speaking versus other complex sounds.

In a block design, voxels not affected by the stimulation should provide a steady set of data points, albeit with some variance due to random and physiological changes not connected with the brain state. Various strategies have been devised for identifying the real, task-related signal changes in the presence of other fluctuations. For example, in comparing the 80 data points in the $\mathrm{ON}$ with the 80 data points in the OFF condition for each voxel, a simple Student's $t$ test may suffice for evaluation of the likelihood that any single voxel has responded. Computation of the relevant statistic at each
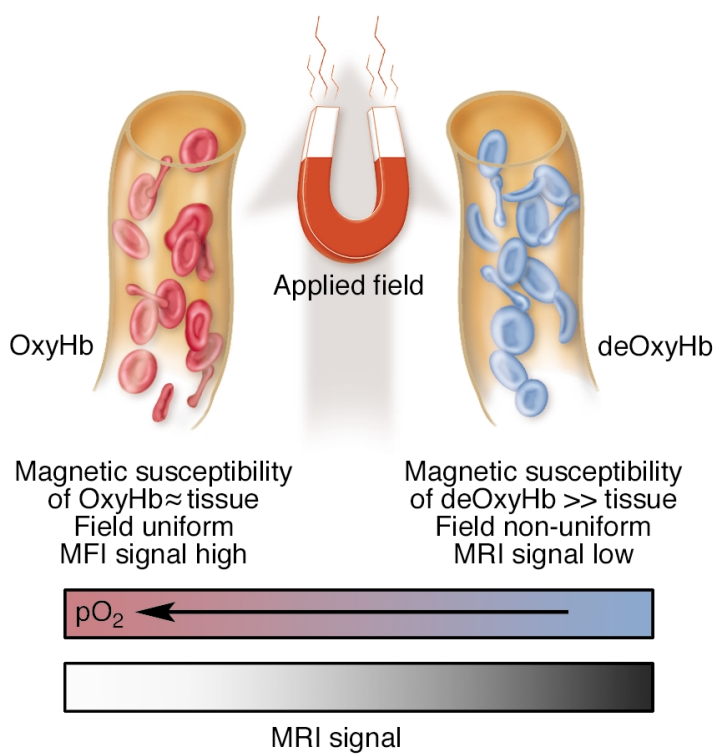

\section{Figure 1}

Schematic illustration of the origins of the BOLD effect in FMRI. While arterial blood is similar in its magnetic properties to tissue, deoxygenated blood is paramagnetic and so induces inhomogeneities within the magnetic field in tissue. These cause the MRI signal to decay faster. Signals from activated regions of cortex increase as the tissue becomes more magnetically uniform. 


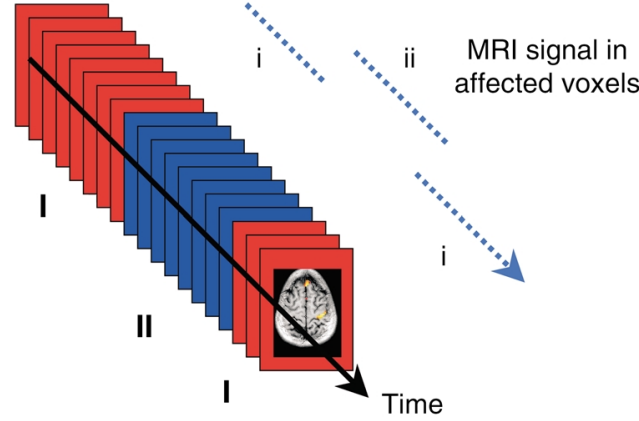

Figure 2

In a block design experiment, sequences of images are acquired in contrasting conditions ( $\mathrm{I}$ and $\mathrm{II}$ ). After acquisition, those voxels whose signals change in synchrony ( $\mathrm{i}$ to ii) with the stimulus or task can be identified.

voxel produces a statistical parametric map of each brain slice. In this map, the voxels that on balance appear to have responded (using some statistical decision criterion, such as $P<0.05)$ are portrayed as "activated". For quantitative comparisons between regions, the percentage signal change is often used, but statistical maps are generally used for evaluation of whether changes are reliable. As with any statistical analysis, positive findings should be couched in probabilities, and there is, therefore, some arbitrariness about the threshold criteria used for defining active regions. This is exacerbated by the fact that within a single image there may be over 10,000 voxels, so that a large number of statistical comparisons are necessarily performed simultaneously. Correcting for this issue of multiple comparisons complicates the assessment of reliability and significance, and at present there are no universally accepted strategies for rigorous analysis of data. The presence of variations that are not task related - for example, from patient motion, respiration, and cardiac pulsations decreases the reliability of fMRI data, and much ongoing research aims to reduce these effects.

The second type of experiment in common use uses transient stimuli that replicate those used in so-called event-related paradigms that have been successfully exploited to record event-related potentials (ERPs) in electrophysiological studies. For testing of many cognitive functions, event-related studies offer a greater variety of more powerful experimental designs. For example, in electrophysiology, a well-known ERP is the P300, which denotes a positive $(\mathrm{P})$ electrical potential that is measurable on the scalp approximately $300 \mathrm{~ms}$ after the detection of an "oddball" event, such as a target stimulus that occurs infrequently and at random within an otherwise regular sequence of stimuli (e.g., a change in a sequence of identical tones) (6). P300s are routinely recorded for diagnostic applications in neurology and psychiatry, and their amplitude and latency are affected by various disorders. If the same set of stimuli is presented with an MRI machine, a transient hemodynamic response is also elicited by each oddball. By averaging of the responses to several "epochs," voxels that show transient fMRI signal changes can be identified. Note that in such an example, although the electrical activity elicited by the oddball stimulus peaks at $300 \mathrm{~ms}$ after the event and lasts for substantially less than 1 second, the corresponding BOLD signal may not peak until several seconds later and endures for much longer. Images recorded at multiple time points after the oddball will portray the time course of the blood flow changes produced by the neural activity (Figure 3 ).

fMRI has found applications in both clinical and more basic neuroscience. Appropriate experiments may now be designed to address specific hypotheses regarding the nature of the distributed systems responsible for
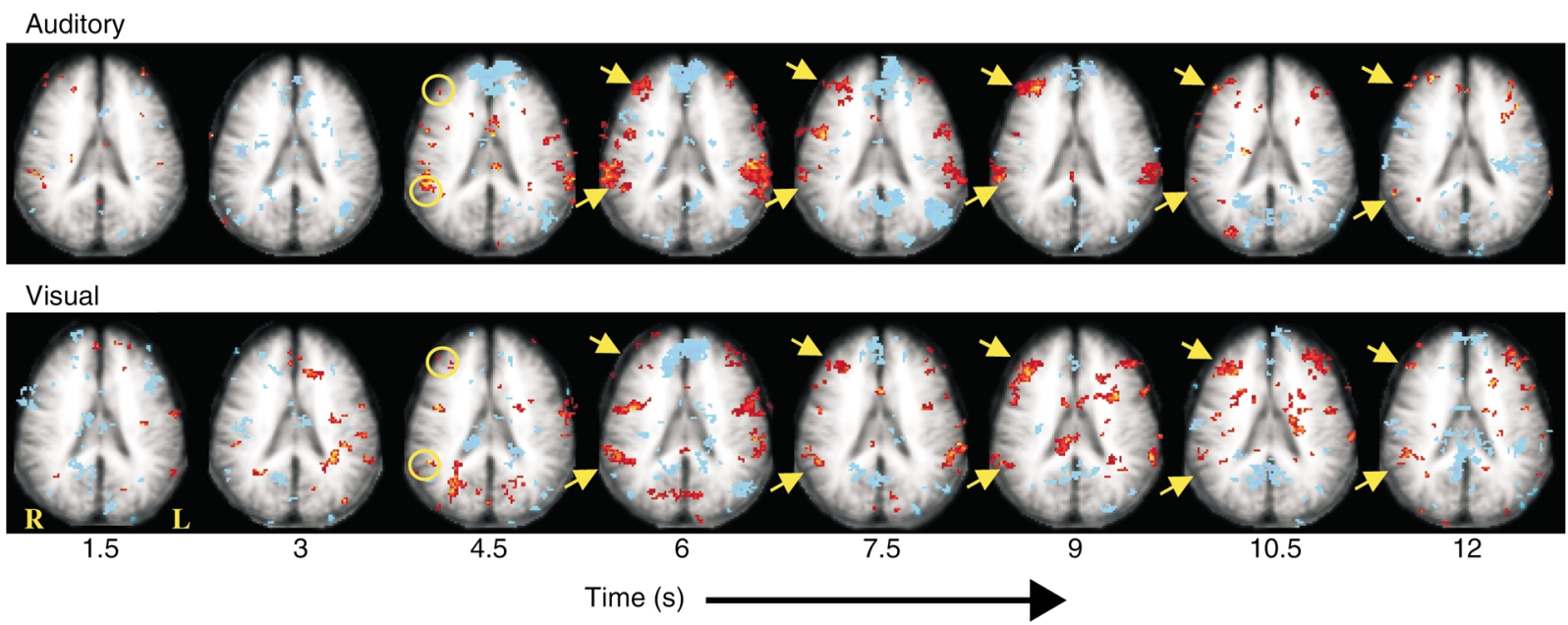

Figure 3

The average time course of the responses of one slice of the brain to oddball stimuli. Each image (left to right) represents the average time course of the response separated by 1.5 seconds, starting at the time of an auditory (top) or a visual (bottom) oddball stimulus. Various regions activate, and some of these activations are independent of the modality of the stimulus. The yellow arrows indicate activation in the middle frontal and supramarginal gyri. Adapted from ref. 18. 


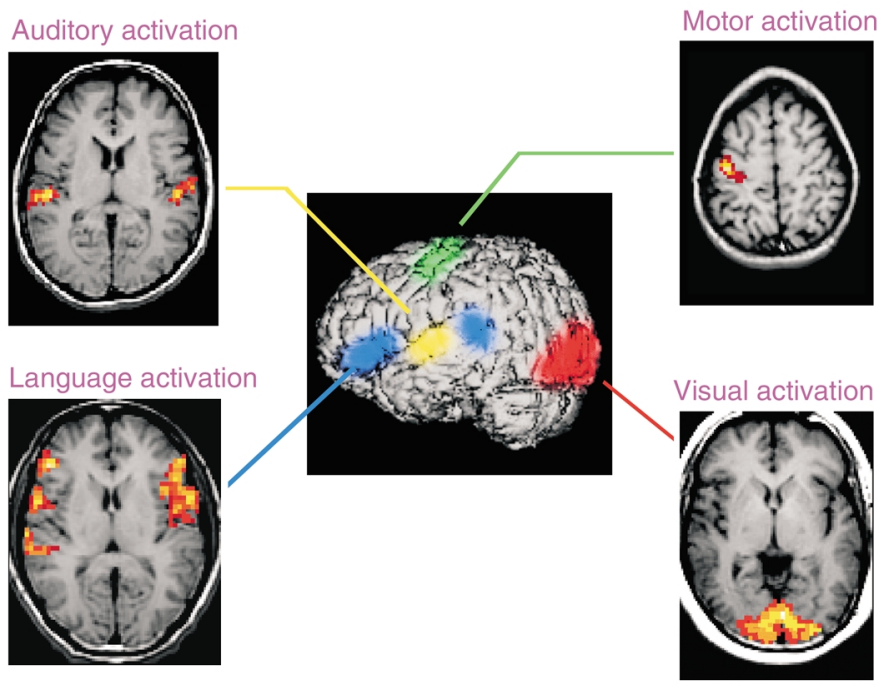

Figure 4

For surgical planning, a series of simple tasks (e.g., auditory, visual, motor, and language tasks) may be performed in a sequence to identify critical functional areas. These may then be superimposed on high-resolution anatomic images.

losses of cortical functions in degenerative disorders such as Alzheimer disease (8). In psychiatry, fMRI is being exploited to delineate the neurobiological bases of various cognitive deficits and aberrant behaviors. For these studies, more sophisticated tasks have been developed that require more subtle or specific cognitive responses. Many studies build on the tasks that have been developed over many years in neuropsychological testing of subjects for the identification of different traits or pathologies. Consider

various functional responses. For clinical applications, simple mapping of critical sensory and motor functions can be readily performed with subjects lying in the bore of a magnet where they perform simple tasks or experience sensory stimuli in blocks. This is the primary approach for evaluations of the brains of patients prior to neurosurgery (e.g., for the treatment of temporal lobe epilepsy or arterio-venous malformations) or radiation therapy. In several centers, standard protocols have been developed that permit the efficient mapping of auditory, visual, motor, and language areas to inform surgeons of the positions of critical functional areas (Figure 4). fMRI data can readily be integrated with image-guided neurosurgical procedures. The identification of eloquent cortex and the hemispheric dominance of language can be performed using fMRI rather than by invasive procedures such as the Wada test (7).

In neurological applications, $\mathrm{AMRI}$ may have a role in studies of patients recovering from stroke, as well as in understanding the extent of occult or asymptomatic the classic Stroop test, in which subjects are required to name the color of various words printed in a colored font. In this test, subjects are instructed explicitly to suppress the (automatic) response of reading the words, and instead to identify only the color of the font. The words displayed may also be the names of the colors of the fonts used, with the word printed sometimes in the congruent color (e.g., "RED" printed in red) and sometimes in an incongruent color (e.g., "RED" printed in blue) (9). Given the propensity to read the word despite the instruction to name only the color of the font, there is a well-documented attentional conflict when the word and the color are incongruent, leading in behavioral studies to a longer reaction time in arriving at the correct color identification. This is the Stroop effect, and it involves several elements of attention and task monitoring as well as correction of automatic responses. In an event-related Stroop test performed in the magnet, a series of congruent word-color pairs may be presented, and incon-
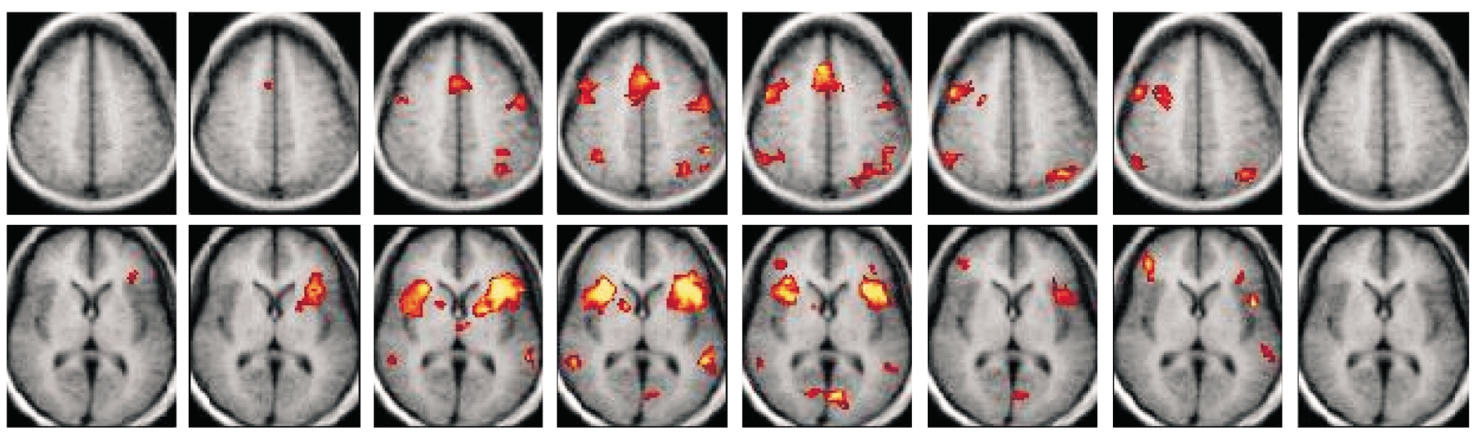

Time after incongruent word

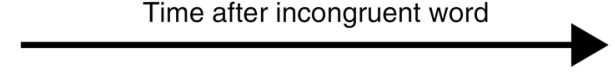

Figure 5

The average time course of the responses of two slices of the brain in the event-related Stroop task. Each image was acquired 1.65 seconds after the previous image, starting at the time of the incongruent word-color pair. Peak activities are apparent in the BOLD response 5-7 seconds after the event. A widespread pattern of activity is seen in both frontal and posterior regions, triggered by the Stroop effect. Adapted from ref. 11. 

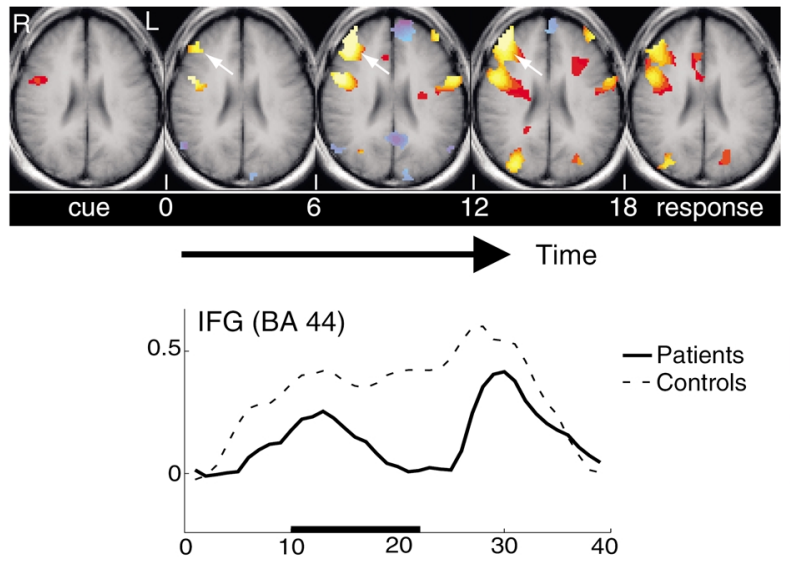

Figure 6

fMRI responses in prefrontal cortex during the rehearsal period of a verbal working memory task. Sustained BOLD effects are seen in normal subjects during the period 6-18 seconds after a series of words is encoded, but patients with schizophrenia fail to maintain this activity in inferior frontal gyrus (IFG), as shown in the lower time course. BA, Brodmann's area.

gruent words can then be interspersed at random. At the appearance of each incongruent word, a set of brain areas begins to activate. Figure 5 shows the time course of these activations; clearly they depict the recruitment and subsequent recovery of multiple brain regions in one or more well-defined networks (10). Stroop tests are one of many such psychological tests that may be used to discriminate various subject groups. In fMRI, the patterns of activation revealed by the Stroop test are different, for example, for subjects with depression and disorders such as pathological gambling, and these differences may be quantified.

One aim of such tests may be to more accurately identify specific differences and deficits, as well as potentially to monitor the effects of treatments or interventions. An example is the use of fMRI to demarcate subgroups of subjects broadly diagnosed with schizophrenia based on characteristic deficits in specific cognitive domains. For example, in fMRI studies, some subjects demonstrate a failure to activate specific frontal regions during a verbal working memory task (11). In this test they are presented with sequences of words to rehearse and to remember for several seconds prior to a simple test of word order. Normal subjects show robust activation in prefrontal cortex throughout the rehearsal period, whereas patients may show muchdiminished and shorter-lived activation over this interval (Figure 6). However, in some subjects the pattern of activation appears to normalize following several weeks of appropriate therapy in which the underlying skills required for this task are emphasized (12). Similar documentation of the effects of therapy or learning have been achieved by several groups in different domains, so that the ability of fMRI to demonstrate the apparent plasticity of the brain is clear. The effects of pharmacologic interventions on cognitive functions may also be revealed via patterns of activation within the brain. For example, acute effects of intravenous administration of ketamine on the circuits activated during P300 stimuli have been quantified. Longer-term changes have also been shown; for example, fMRI studies of language have demonstrated cognitive effects produced by hormone replacement treatments with estrogen (13).

Among the most important applications of $\mathrm{PMRI}$ is the study of neurodevelopment and disorders associated with children, where the safety and noninvasive nature of MRI are paramount. For example, fMRI has been used to demonstrate the failure of autistic individuals to recruit the cortical substrate (which includes the fusiform gyrus) used for face processing by normal subjects, providing an anatomic basis for interpreting the lack of affect that such subjects demonstrate when confronted with human faces (14). In developmental dyslexia, fMRI has been used to identify deficits in specific posterior circuits involving the angular gyrus that account for reading disability (15), and activations within these circuits have been shown to correlate well with reading skill (16) and to respond to educational interventions that focus on specific language and reading skills. fMRI may have an important role in evaluating the benefits of specific learning strategies and other interventions.

An important area of further development is the application of advanced data analysis techniques and modeling in order to interpret fMRI results. For mapping of single critical functions (e.g., for neurosurgical planning), a simple but accurate map of activated voxels may be sufficient, but for many other purposes, a knowledge of where activity occurs may not advance our understanding of brain function significantly. However, greater insights into the neural basis of behavior may be obtained by examination of the manner in which regional activities vary with behavioral or other performance or physiological measures, or across tasks, or among subjects within a group, and between groups. To quantify these covariations, more advanced mathematical techniques have been developed, many of which resemble the multivariate statistical methods employed to derive relationships among variables in nonimaging data (17). By such approaches, the relationship of fMRI activity to specific behavioral measures, and the connectivity between different brain regions, may be derived. Such methods have proven especially helpful in extracting new information on brain systems involved in complex responses from simple maps of activation acquired during different conditions.

fMRI has several limitations. The use of fast imaging reduces the spatial resolution to a few millimeters, somewhat worse than conventional MRI. The BOLD effect is small, and thus the sensitivity is limited, so that fMRI experiments require multiple samplings of brain responses. The temporal resolution is poor and is limited by the nature of the hemodynamic response. Furthermore, the reliability is reduced when there are significant subject motions or physiologically related 
variations. The origins and influence of various sources of such variance are not yet completely understood. For example, the importance of variations in the blood levels of everyday substances (such as caffeine, nicotine, and glucose), or of hormones (such as estrogen), all of which are likely to affect the BOLD signal, is not well documented. Aging and impaired cerebrovascular supply are also likely to affect the magnitude of the BOLD response. The BOLD effect is an indirect measure of neural activity, and there are couplings at different stages - for example, between electrical activity and metabolic flux, and between neurotransmitter release and energy supply - that are not well understood. Nonetheless, fMRI signals are not simply an epiphenomenon of neural activity, and they clearly are sensitive to subtle changes in the state of the brain, as well as to the effects of learning, of expertise, and of various treatments. fMRI provides an accurate and painless method for mapping of critical functions and likely has a much larger role to play in the management of clinical patients for diverse disorders in the future.

1. Pauling, L., and Coryell, C.D. 1936. The magnetic properties and structure of hemoglobin, oxyhemoglobin and carbonmonoxyhemoglobin. Proc. Natl. Acad. Sci. U. S. A. 22:210-216.

2. Ogawa, S., Lee, T.M., Kay, A.R., and Tank, D.W. 1990. Brain magnetic resonance imaging with contrast dependent on blood oxygenation. Proc. Natl. Acad. Sci. U. S. A. 87:9868-9872.

3. Robson, M., Dorosz, J.L., and Gore, J.C. 1998. Measurements of the temporal fMRI response of the human auditory cortex to trains of tones. Neuroimage. 7:185-198.

4. Constable, R.T., McCarthy, G., Allison, T., Anderson, A.W., and Gore, J.C.
1993. Functional brain imaging at $1.5 \mathrm{~T}$ using conventional gradient echo MR imaging techniques. Magn. Reson. Imaging. 11:451-459.

5. Cohen, M.S., and Weisskoff, R.M. 1991. Ultra-fast imaging. Magn. Reson. Imaging. 9:1-37.

6. Sutton, S., Braren, M., Zubin, J., and John, E.R. 1965. Evoked potential correlates of stimulus uncertainty. Science. 150:1187-1188.

7. Schlosser, M., Aoyagi, N., Fulbright, R.K., Gore, J.C., and McCarthy, G. 1998. Functional MRI studies of auditory comprehension. Hum. Brain Mapp. 6:1-13.

8. Smith, C.D., et al. 1990. Altered brain activation in normal subjects at risk for Alzheimer's disease. Neurology. 53:1391-1396.

9. Stroop, J.R. 1935. Studies of interference in serial verbal reactions. J. Exp. Psychol. 18:643-662.

10. Leung, H.C., Skudlarski, P., Gatenby, J.C., Peterson, B.S., and Gore, J.C. 2000. An event-related functional MRI study of the stroop color word interference task. Cereb. Cortex. 10:552-560.

11. Stevens, A.A., Goldman-Rakic, P.S., Gore, J.C., Fulbright, R.K., and Wexler, B.E. 1998. Cortical dysfunction in schizophrenia during auditory word and tone working memory demonstrated by functional magnetic resonance imaging. Arch. Gen. Psychiatry. 55:1097-1103.

12. Wexler, B.E., Anderson, M., Fulbright, R.K., and Gore, J.C. 2000. Preliminary evidence of improved verbal working memory performance and normalization of task-related frontal lobe activation in schizophrenia following cognitive exercises. Am. J. Psychiatry. 157:1694-1697.

13. Shaywitz, S.E., et al. 1999. Effect of estrogen on brain activation patterns in postmenopausal women during working memory tasks. JAMA. 281:1197-1202.

14. Schultz, R.T., et al. 2000. Abnormal ventral temporal cortical activity among individuals with autism and Asperger syndrome during face discrimination. Arch. Gen. Psychiatry. 57:331-340.

15. Shaywitz, S.E., et al. 1998. Functional disruption in the organization of the brain for reading in dyslexia. Proc. Natl. Acad. Sci. U. S. A. 95:2636-2641.

16. Shaywitz, B.A., et al. 2002. Disruption of posterior brain systems for reading in children with developmental dyslexia. Biol. Psychiatry. 52:101-110.

17. Mencl, W.E., et al. 2000. Network analysis of brain activations in working memory: behavior and age relationships. Microsc. Res. Tech. 51:64-74.

18. Stevens, A.A., Skudlarski, P., Gatenby, J.C., and Gore, J.C. 2000. Eventrelated $\mathrm{fMRI}$ of auditory and visual oddball task. Magn. Reson. Imaging. 18:495-502. 